\title{
Future directions for identifying the neural correlates of consciousness
}

\author{
Kristian Sandberg, Stefan Frässle and Michael Pitts
}

In their interesting and thorough Review (Neural correlates of consciousness: progress and problems. Nat. Rev. Neurosci. 17, 307-321 $(2016))^{1}$, Koch and colleagues argue that the fronto-parietal network is not directly related to consciousness as was previously thought. Instead, they argue that consciousness is related to what they call the 'posterior cortical hot zone' as well as to differentiated and integrated information (information complexity). Readers of the Review will not be surprised to hear that we, in general, welcome this paradigm shift. However, we were surprised by the lack of focus on future directions, and we are concerned that the newly coined term 'posterior cortical hot zone' could be misleading in some cases.

Koch and colleagues acknowledge that their Review is based mainly on visual experiments, which may bias their conclusions. Along the lines of what we have previously $\operatorname{argued}^{2-4}$, the authors emphasize the role of sensory areas as prime candidates for neural correlates of consciousness (NCC). However, although visual conscious experience certainly appears to be related to activity in the posterior cortical regions, this is probably not the case for conscious experiences within other sensory domains where perceptual processing does not take place in the posterior cortex - the gustatory cortex, for instance, is located partly in the frontal lobe. For this reason, the term 'posterior cortical hot zone' may turn out to be inaccurate, and our focus in the search for correlates of sensory consciousness should lie on the cortical areas involved in the processing of the respective stimuli.

Although activity in sensory areas appears to be a main candidate for the correlate of conscious experience, it is still unclear which characteristics of this activity are related to consciousness, how this activity relates to the anatomy of these areas ${ }^{5,6}$, what the underlying mechanisms are and how this is connected to information complexity. In particular, the link between information complexity and a sensory or posterior cortical hot zone needs further clarification. At present, the two do not appear to be directly related, and some potentially paradoxical observations have been made: for instance, the perturbational complexity index, a transcranial magnetic stimulation-electroencephalographic-based measure of complexity, is equally predictive of conscious states whether it is measured at Brodmann area 8 (BA8; frontal lobe) or at BA19 (occipital lobe) ${ }^{7}$.

Koch and colleagues briefly mention the visual awareness negativity $(\mathrm{VAN})^{8}$, which is thought to originate from a number of posterior cortical sources ${ }^{3,9}$, as a prime electrophysiological correlate of visual consciousness. Although we agree that VAN shows promise as a potential neural correlate of consciousness, we note that many questions remain to be tackled. Indeed, do different sources of the VAN relate to different aspects of phenomenology? Moreover, is the absolute amplitude of a single VAN source a better predictor of conscious experience than its relative amplitude compared to neighbouring, competing sites? Finally, which VAN sources directly index awareness and which reflect selective attention processes?

The Review by Koch et al. places heavy emphasis on the identification of the neuroanatomical locations of NCC. It is entirely possible, however, that localizing NCC is less important than determining the particular computations carried out by the relevant interconnected circuits. What are these computations? Which circuits are involved? And why?

Overall, addressing the questions outlined in this Correspondence will provide us with deeper insight into the role of posterior (and anterior) sensory areas in conscious phenomenology and will bring us closer to unravelling the true NCC.

Kristian Sandberg is at the Cognitive Neuroscience Research Unit, Center of Functionally Integrative Neuroscience, Hammel Neurorehabilitation and Research Centre, Danish Neuroscience Center, Aarhus University Hospital, 8000 Aarhus, Denmark.

Stefan Frässle is at the Translational Neuromodeling Unit, Institute for Biomedical Engineering, University of Zurich and ETH Zurich, Zurich 8032, Switzerland.

Michael Pitts is at the Department of Psychology, Reed College, Portland, Oregon 97202-8199, USA.

Correspondence to K.S krissand@rm.dk

doi: $10.1038 /$ nrn. 2016.104 Published online 28 Jul 2016

1. Koch, C., Massimini, M., Boly, M. \& Tononi, G. Neural correlates of consciousness: progress and problems. Nat. Rev. Neurosci. 17, 307-321 (2016).

2. Frässle, S., Sommer, J., Jansen, A., Naber, M. \& Einhäuser, W. Binocular rivalry: frontal activity relates to introspection and action but not to perception. J. Neurosci. 34, 1738-1747 (2014).

3. Sandberg, K. et al. Early visual responses predict conscious face perception within and between subjects during binocular rivalry. J. Cogn. Neurosci. 25 , 969-985 (2013).

4. Pitts, M. A., Martínez, A. \& Hillyard, S. A. Visual processing of contour patterns under conditions of inattentional blindness. J. Cogn. Neurosci. 24, 287-303 (2012).

5. Schwarzkopf, D. S., Song, C. \& Rees, G. The surface area of human $\mathrm{V} 1$ predicts the subjective experience of object size. Nat. Neurosci. 14, 28-30 (2011).

6. Kanai, R., Carmel, D., Bahrami, B. \& Rees, G. Structural and functional fractionation of right superior parietal cortex in bistable perception. Curr. Biol. 21, R106-R107 (2011).

7. Casali, A. G. et al. A theoretically based index of consciousness independent of sensory processing and behavior. Sci. Transl Med. 5, 198ra105 (2013).

8. Wilenius-Emet, M., Revonsuo, A. \& Ojanen, V. An electrophysiological correlate of human visual awareness. Neurosci. Lett. 354, 38-41 (2004).

9. Koivisto, M. \& Revonsuo, A. Event-related brain potential correlates of visual awareness. Neurosci. Biobehav. Rev. 34, 922-934 (2010).

Competing interests statement

The authors declare no competing interests. 